\title{
Evidence for a Crossover in the Frequency Dependence of the Acoustic Attenuation in Vitreous Silica
}

\author{
C. Masciovecchio, ${ }^{1}$ G. Baldi, ${ }^{2}$ S. Caponi, ${ }^{4}$ L. Comez, ${ }^{3,4}$ S. Di Fonzo, ${ }^{1}$ D. Fioretto, ${ }^{3,4}$ A. Fontana, ${ }^{2,4}$ A. Gessini, ${ }^{1}$ \\ S. C. Santucci, ${ }^{1,3}$ F. Sette, ${ }^{5}$ G. Viliani, ${ }^{2,4}$ P. Vilmercati, ${ }^{1}$ and G. Ruocco ${ }^{6,4}$ \\ ${ }^{1}$ Sincrotrone Trieste, strada statale $14 \mathrm{~km}$ 163.5, 34012 Basovizza, Trieste, Italy \\ ${ }^{2}$ Dipartimento di Fisica, Universitá di Trento, 38050 Povo, Trento, Italy \\ ${ }^{3}$ Dipartimento di Fisica, Universitá di Perugia, 06123 Perugia, Italy \\ ${ }^{4}$ CRS SOFT-INFM-CNR, at Universitá di Roma La Sapienza, 00185 Roma, Italy \\ ${ }^{5}$ European Synchrotron Radiation Facility, BP 220, 38043 Grenoble, France \\ ${ }^{6}$ Dipartimento di Fisica, Universitá di Roma LaSapienza, 00185 Roma, Italy
}

(Received 30 August 2005; published 18 July 2006)

\begin{abstract}
We report measurements of the sound attenuation coefficient in vitreous silica, for sound waves of wavelength between 50 and $80 \mathrm{~nm}$, performed with the new inelastic UV light scattering technique. These data indicate that in silica glass a crossover between a temperature-dependent (at low frequency) and a temperature-independent (at high frequency) acoustic attenuation mechanism occurs at $Q \approx 0.15 \mathrm{~nm}^{-1}$. The absence of any signature in the static structure factor at this $Q$ value suggests that the observed crossover should be associated with local elastic constant fluctuations.
\end{abstract}

PACS numbers: 61.43.Fs, 63.50.+x

The sound attenuation in disordered materials and its frequency and wavelength dependence are the result of the interplay between two physical mechanisms: one is due to the anharmonicity of the interparticle interactions, and the other to the structural disorder.

The anharmonic attenuation of an acoustic sound wave, identified by its wavelength $\lambda$, frequency $\Omega$, and wave vector $Q=2 \pi / \lambda$, is characterized by a specific, temperature-dependent, relaxation time $\tau_{r}$ [1]. At low frequency $\left(\omega \tau_{r}<1\right)$ this process dominates the sound absorption through mechanisms such as, e.g., the Akhiezer mechanism [2,3]. Accordingly, the sound attenuation coefficient, as measured by the broadening $\Gamma$ of the Brillouin peak in the dynamic structure factor $S(Q, \omega)$, scales as $\omega^{2}$ and $Q^{2}$. At high frequency $-\omega \tau_{r}>1$, i.e., $Q>Q_{r}=$ $1 / v \tau_{r}$, where $v$ is the sound velocity, the anharmonic attenuation becomes frequency independent [1-3].

The sound attenuation associated with topological disorder gives rise to a steeper $Q$ dependence of $\Gamma(Q)$. If Rayleigh scattering is responsible for this attenuation, $\Gamma \propto$ $Q^{4}$ is expected for wavelengths larger than the typical defects size $\xi=2 \pi / Q_{R}$. For $Q>Q_{R}$, when the Rayleigh scattering regime is abandoned, one expects that $\Gamma(Q)$ is no longer $\propto Q^{4}$. Experimentally, for $Q$ larger than $1 \mathrm{~nm}^{-1}$, all glasses studied so far show $\Gamma \propto Q^{x}$, with $x$ very close to $2[4,5]$.

This scenario can be summarized by a three-regime behavior of $\Gamma(Q)$ : (i) at low $Q, \Gamma(Q)$ is determined by anharmonic processes, and $\Gamma(Q) \propto Q^{2}$ up to a first (temperature-dependent) crossover $Q_{r}=1 / v \tau_{r}$; (ii) an intermediate regime, where the $Q$ dependence of $\Gamma(Q)$ is determined by the system dependent strengths of anharmonicity and structural disorder processes; (iii) a high- $Q$ regime, where $\Gamma(Q)$ is determined by the topological disorder and $\Gamma(Q) \propto Q^{2}$ with a temperature-independent co- efficient. This picture is highly debated because it critically depends on the location of $Q_{r}$ and $Q_{R}$ in different glasses. In densified $v-\mathrm{SiO}_{2}$, for example, the crossover $Q_{R}$ has been hypothesized to be around $2 \mathrm{~nm}^{-1}$ [6].

In the most studied case of vitreous silica, similarly to what happens in many other glasses, both $Q_{r}$ and $Q_{R}$ belong to a $Q$ region which is not directly accessed by traditional scattering probes. In the case of $v-\mathrm{SiO}_{2}$, clear evidence is reported for the low- and high- $Q$ quadratic behaviors of $\Gamma(Q)$, using Brillouin light scattering (BLS) $[7,8]$ and inelastic x-ray scattering (IXS) [9], respectively. Of fundamental importance is the observation that the two quadratic behaviors do not have the same coefficient, and therefore do not extrapolate one onto the other. This provides some evidence for the presence of an intermediate regime $[8,10]$.

In this Letter, we report the characterization of this intermediate regime in $\mathrm{SiO}_{2}$. We report measurements of the dynamic structure factor of vitreous silica using the newly developed inelastic ultraviolet scattering (IUVS) technique [11]. This probe, using a photon energy approximately 4 times larger than that usually employed in BLS experiments, allows one to study sound waves up to a momentum transfer region of $0.14 \mathrm{~nm}^{-1}$. Our experimental results show the transition between the two quadratic behaviors and indicate a value of $Q_{R} \approx 0.15 \mathrm{~nm}^{-1}$.

The experiment was performed at the IUVS beam line at the Elettra Synchrotron Radiation Laboratory in Trieste [11], with incident photon energies between 5 and $8.2 \mathrm{eV}$; we performed experiments on several vitreous silica samples. The results from different samples appeared to be indistinguishable, and in all cases the photon absorption was measured to be negligible in the investigated energy region, thus indicating that the contribution to the width of Brillouin lines coming from the finite penetration 
depth could be neglected up to this energy. The spectra were taken in the range $\pm 1 \mathrm{meV}$ with a CCD cooled detector (noise of $1 \mathrm{el} / \mathrm{pixel} / \mathrm{h}$ ). The spectra were obtained by integrating over a strip $0.26 \mathrm{~mm}$ long orthogonal to the energy dispersion direction. The instrumental total relative energy resolution was about $5 \times 10^{-5}$. At the lowest incident photon energies, the total integration time for each spectrum was $1 \mathrm{~h}$, giving rise to a total inelastic intensity of $\approx 400$ counts/channel.

A set of representative IUVS spectra taken at different momentum transfers and at $T=300 \mathrm{~K}$ is reported in Fig. 1: the Brillouin peak shift and broadens as the momentum transfer increases. In order to extract quantitative information on the $Q$ dependence of the parameters describing the peaks and, in particular, the sound attenuation coefficient, the spectra were fitted by a model function made up by the convolution of the experimental resolution function with $S(Q, \omega)$ represented by the sum of a $\delta$ function and a damped harmonic oscillator [12,13] for the elastic and inelastic contributions, respectively:

$$
S(Q, \omega)=A \delta(\omega)+B \frac{\Omega^{2}(Q) \Gamma(Q)}{\left[\omega^{2}-\Omega^{2}(Q)\right]^{2}+\omega^{2} \Gamma^{2}(Q)},
$$

where $A$ and $B$ are intensity parameters. As an example of the results of the fitting procedure, in Fig. 2 we show the spectra at $Q=0.095 \mathrm{~nm}^{-1}, Q=0.127 \mathrm{~nm}^{-1}$, and $Q=$ $0.138 \mathrm{~nm}^{-1}$. The values derived from the fit for $\Omega(Q)$ show a linear dispersion with $Q$ and a slope corresponding to a sound velocity $v=5950 \mathrm{~m} / \mathrm{s}$, consistent with previous measurements in the low- $Q$ (BLS) and high- $Q$ (IXS)

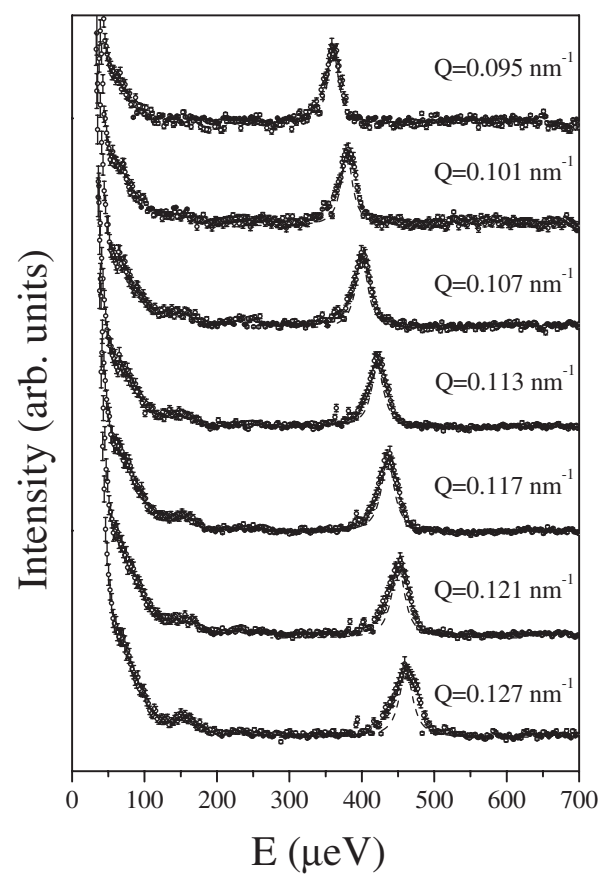

FIG. 1. Brillouin spectra (Stokes side) of vitreous silica (open circles with statistical $\pm 1 \sigma$ error bars) at the selected $Q$ values indicated in the figure. The dashed curves aligned with the Brillouin peaks are the instrumental resolution functions. regions. The values of the widths $\Gamma(Q)$ are displayed in Fig. 3 as a function of $Q$ in the examined $Q$ range. In this figure we also report as dot-dashed and dot-dot-dashed lines the extrapolated quadratic behavior of $\Gamma(Q)=$ $D Q^{2}$, as measured at low $Q$ by BLS $\left(D_{\mathrm{BLS}}=\right.$ $\left.0.45 \mathrm{meVnm}^{2}\right)$ and at high $Q$ by IXS $\left(D_{\mathrm{IXS}}=\right.$ $0.98 \mathrm{meV} \mathrm{nm}^{2}$ ). The difference between $D_{\mathrm{BLS}}$ and $D_{\mathrm{IXS}}$, as well as its physical origin, is the central issue of the present work.

From Fig. 3, it can be observed that the low- $Q$ IUVS values of $\Gamma(Q)$ are superimposed to the extrapolation of the BLS data, while, at high $Q, \Gamma(Q)$ exhibits a clear departure from the BLS behavior: In this high- $Q$ region, $\Gamma(Q)$ rises towards the curve extrapolated from the IXS data.

In order to compare the present data to measurements performed in different $Q$ ranges, in Fig. 4 we report in loglog scale the parameter $\Gamma(Q)$ for room-temperature vitreous silica as measured by IUVS (present work), IXS [9], and BLS $[4,7,8]$. As previously discussed, both the low- $Q$ (BLS, $Q<4 \times 10^{-2} \mathrm{~nm}^{-1}$ ) and the high- $Q$ (IXS, $Q>$ $1 \mathrm{~nm}^{-1}$ ) data show a $Q^{2}$ dependence of $\Gamma(Q)$, described by the dot-dot-dashed and dot-dashed lines, respectively, but with coefficients which differ by approximately a factor of 2 . Therefore, the literature data identify a $Q$ range between $\approx 0.05$ and $1 \mathrm{~nm}^{-1}$ where a transition between the two regimes must take place. The new IUVS data reported in the present Letter partially cover this transition

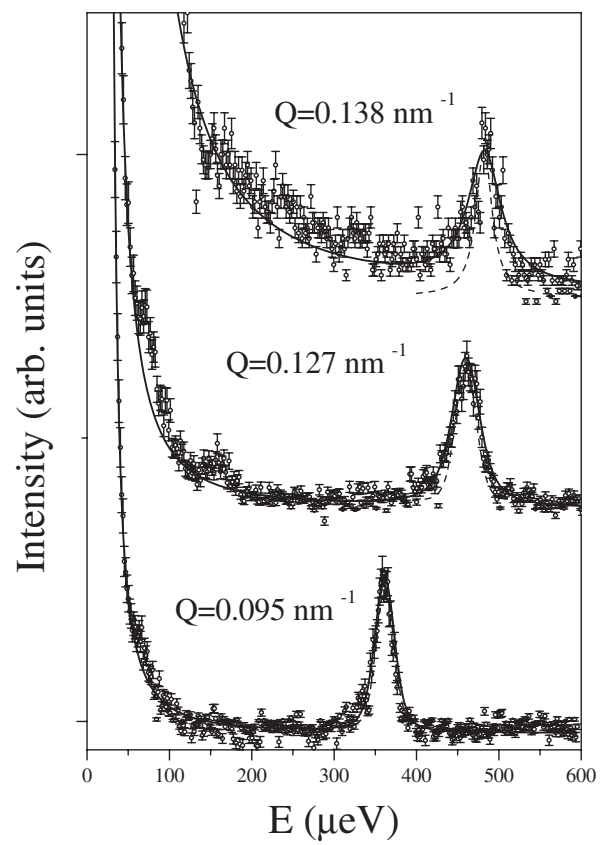

FIG. 2. Brillouin spectra (Stokes side) of vitreous silica (open circles with statistical $\pm 1 \sigma$ error bars) at $Q=0.095 \mathrm{~nm}^{-1}, Q=$ $0.127 \mathrm{~nm}^{-1}$, and $Q=0.138 \mathrm{~nm}^{-1}$. The solid lines superimposed on the experimental data are the best fitted line shapes, while the dashed curves aligned with the Brillouin peaks are the instrumental resolution functions. The tail at low energies, visible in the spectrum at $Q=0.138 \mathrm{~nm}^{-1}$, is due to the instrumental elastic line. 


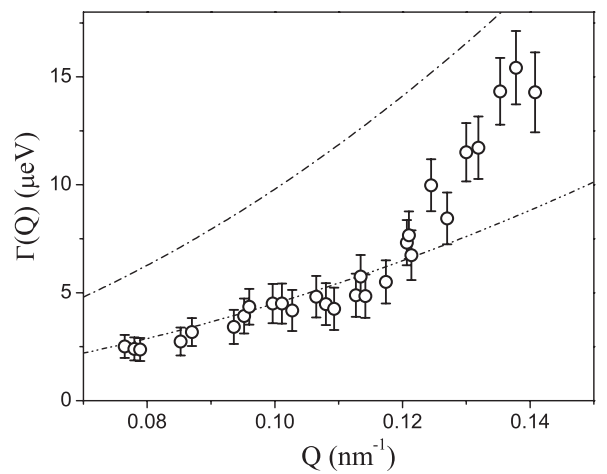

FIG. 3. Width of the Brillouin peaks of room-temperature silica, as a function of the momentum transfer, as extracted by fitting IUVS data to Eq. (1). The dot-dashed and dot-dot-dashed lines correspond to the extrapolation of the quadratic behaviors measured at low $Q$ using BLS (Refs. [7,8]) and at high $Q$ using IXS (Ref. [5]).

region and show (i) the departure from the low- $Q$ quadratic dependence and (ii) the sharp rise towards the high- $Q$ quadratic dependence that, however, is not fully reached in the investigated $Q$ range.

Some measurements of acoustic attenuation on silica thin films in this transition region, performed with the tunneling junctions (TJs) technique at temperatures around $1 \mathrm{~K}$, are indeed found in the literature [14]. These data will not be considered in the following discussion because they do not seem to be directly comparable with our measurements on bulk $v-\mathrm{SiO}_{2}$ samples. It is worth noting that the TJ data are dominated by uncontrolled nonequilibrium and nonlinear effects [15] and that their interpretation is highly controversial, as similar independent measurements give results which differ up to a factor of 5 [16].

In principle, the presence of photons absorption in the sample, and the consequent exponentially decaying envelope of the light intensity, gives rise to an additional broadening of the Brillouin peak because of the ill definition of the wave vector $Q$. This additional spurious broadening is $\Delta \Gamma \approx 2(k / n) \hbar \Omega(Q)$. Here $n$ and $k$ are the real and imaginary part of the refractive index, respectively. At a photon energy $E=8.2 \mathrm{eV}$ - the higher energy considered in this work - the value $n$ and $k$ of vitreous silica are $n=1.73$ and $k=4.63 \times 10^{-4}$ [17]. Therefore at this energy (that in the scattering geometry employed in the present experiment correspond to $Q=0.144 \mathrm{~nm}^{-1}$ ) the contribution to the width of the Brillouin peaks turns out to be $\Delta \Gamma \approx$ $0.3 \mu \mathrm{eV}$, negligible with respect to the natural broadening $(\Gamma \approx 10 \mu \mathrm{eV})$. The upper inset of Fig. 4 shows the measured Brillouin width (open circles) compared to that expected on the basis of the values of $n$ and $k$ from literature (open squares) [17] and from a direct measurement (open diamonds) performed on the same samples used for the UV-BLS experiment at the Elettra beam line BADElph [18]. These data lead one to discard the possibility that the measured width is an artifact associated with photon absorption.

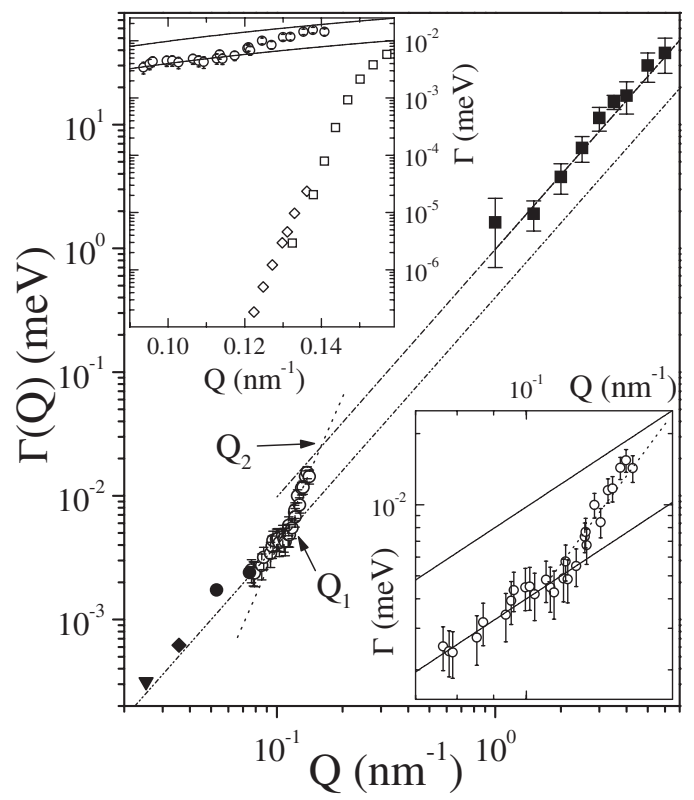

FIG. 4. $\Gamma(Q)$ from different data sets: Circles, IUVS (this work); triangles and diamonds, visible BLS (Refs. [7,9]); solid circles, BLS data with $266 \mathrm{~nm}$ photons (Ref. [8]); squares, IXS (Refs. [5,9]). The dotted line corresponds to a $Q^{4}$ law while $Q_{1}$ and $Q_{2}$ are the crossing point discussed in the text. The lower inset shows an enlargement of the newly investigated $Q$ region, where the departure from the low- $Q$ quadratic law is found. The upper inset shows in a log-lin scale the $\Gamma(Q)$ from this work (open circles) compared to that expected on the basis of the values of $n$ and $k$ from literature (open squares) [17] and from a direct measurement (open diamonds) performed on the same samples used for the UV-BLS experiment [18].

The scheme reported in Fig. 4 has two "crossing" points: the first crossover, $Q_{1}$, divides the low-frequency $Q^{2}$ behavior from an higher frequency $Q^{\alpha}$ law, with $\alpha$ compatible with 4 . This crossover has never been observed before. The second crossover, $Q_{2}$, corresponds to a subsequent (in the direction of increasing $Q$ values) bending of the $Q^{\alpha}$ behavior to the $Q^{2}$ law observed in the $\mathrm{THz}$ frequency region by x-rays Brillouin scattering. Although the value of $Q_{2}$ has not been reached in the present experiment, due to the high photon absorption by the sample above $8.3 \mathrm{eV}$, the existence of this crossover at $Q_{2}$ is the simplest way to reconcile the $Q^{\alpha}$ slope observed here with the high-frequency $Q^{2}$ law. The present data provide a value $Q_{1}=0.11 \pm 0.01 \mathrm{~nm}^{-1}$ for $Q_{1}$ and, using a sound velocity $v=5950 \mathrm{~m} / \mathrm{s}, \nu_{1}=100 \pm 10 \mathrm{GHz}$. Moreover, IUVS data allow one to estimate by extrapolation the crossover $Q_{2}$ value as $Q_{2}=0.15 \pm 0.01 \mathrm{~nm}^{-1}$ and $\nu_{2}=$ $140 \pm 10 \mathrm{GHz}$.

Two possible explanations can be invoked for the existence of a crossover at $Q=Q_{2}$. In the first one, the crossover can be interpreted in terms of a characteristic length scale $\left(\xi=2 \pi / Q_{2}\right)$ of elastic constants inhomogeneity of about $40 \mathrm{~nm}$, a value that is surprisingly high and that corresponds to almost 70 nearest $\mathrm{SiO}_{4}$ tetrahedra. In this framework $Q_{2}=Q_{R}$, i.e., the momentum transfer where 
Rayleigh scattering from inhomogeneities is the dominant sound attenuation mechanism. $\xi$ cannot be associated with density inhomogeneities, as no characteristic signature is found in the static structure factor at $Q=Q_{R}$. Besides local density, the propagation of sound waves is controlled also by the local elastic constants, and therefore the obtained result can be explained by inhomogeneities of the local forces on the scale $\xi$. This is consistent with the existence of such correlations as identified by computer simulations [19-21]. In particular, in two recent papers, Tanguy, Leonforte, and collaborators have shown the existence of a characteristic length in the stress correlation function of a model Lennard-Jones glass in two [19] and three [21] dimensions. They found inhomogeneous regions on a length scale of 30 interparticle distances in a $2 \mathrm{D}$ system (particles in a plane), and inhomogeneous regions on a length scale of 23 interparticle distances in a 3D system (particles in the space). Therefore a tentative interpretation for the origin of $\xi$ is to assign this length scale to the scale of force constants' fluctuations.

A second possible interpretation relies on a recently proposed elastic continuum model [22]. Within this model the crossover can be related to the onset of an excess of states with respect to the Debye density of states. Specifically, in this theory, the width of the Brillouin line results to be proportional to the excess of states and this quantity behaves as $Q^{2}$ in the whole Boson Peak energy region and as $Q^{4}$ below a system dependent onset frequency [23].

In conclusion, we have reported an inelastic ultraviolet scattering measurement of the dynamic structure factor of vitreous silica in a momentum transfer region not accessible so far by other spectroscopic techniques. This study has revealed that a crossover exists in the acoustic attenuation of vitreous silica at $Q \approx 0.15 \mathrm{~nm}^{-1}$. This crossover may be associated with the existence of static fluctuations in the elastic constants. Two models have been proposed to account for this behavior. In both models the elastic constant disorder in a homogeneous density medium plays a central role; the difference lies in the correlation length of the disorder. In the first case, this length $\xi$ is large and determines the $Q_{R}$ value. In the second one, $\xi=0$ and the crossover frequency is determined by the variance of the elastic constants distribution.

As a final remark, we note that the present observation leads to a revision of old ideas on the thermal conductivity of glasses [24,25], which were based on the existence of a $Q^{4}$ dependence of the acoustic attenuation in the region just below $Q \approx 1 \mathrm{~nm}^{-1}$.

[1] J. Fabian and P. B. Allen, Phys. Rev. Lett. 82, 1478 (1999).

[2] A. Akhiezer, J. Phys. (Moscow) 1, 277 (1939).
[3] H. J. Maris, in Physical Acoustics, edited by W. P. Mason and R. N. Thurston (Academic, New York, 1971), Vol. VIII.

[4] G. Ruocco et al., Phys. Rev. Lett. 83, 5583 (1999).

[5] C. Masciovecchio et al., Phys. Rev. Lett. 76, 3356 (1996); P. Benassi et al., Phys. Rev. Lett. 77, 3835 (1996); F. Sette et al., Science 280, 1550 (1998); C. Masciovecchio et al., Phys. Rev. Lett. 80, 544 (1998); A. Mermet et al., Phys. Rev. Lett. 80, 4205 (1998); G. Monaco, D. Fioretto, C. Masciovecchio, G. Ruocco, and F. Sette, Phys. Rev. Lett. 82, 1776 (1999); A. Fontana et al., Europhys. Lett. 47, 56 (1999); A. Sokolov et al., Phys. Rev. E 60, R2464 (1999); O. Pilla et al., Phys. Rev. Lett. 85, 2136 (2000); C. Masciovecchio, A. Mermet, G. Ruocco, and F. Sette, Phys. Rev. Lett. 85, 1266 (2000); A. Matic, D. Engberg, C. Masciovecchio, and L. Borjesson, Phys. Rev. Lett. 86, 3803 (2001); A. Matic et al., Europhys. Lett. 54, 77 (2001); G. Ruocco and F. Sette, J. Phys. Condens. Matter 13, 9141 (2001); T. Scopigno et al., J. Chem. Phys. 118, 311 (2003); Phys. Rev. Lett. 92, 025503 (2004); J. Mattsson et al., J. Phys. Condens. Matter 15, S1259 (2003); R. Zorn et al. (to be published).

[6] B. Rufflé, M. Foret, E. Courtens, R. Vacher, and G. Monaco, Phys. Rev. Lett. 90, 095502 (2003).

[7] J. A. Bucaro and H.D. Dardy, J. Appl. Phys. 45, 5324 (1974).

[8] P. Benassi et al., Phys. Rev. B 71, 172201 (2005).

[9] P. Benassi et al., Phys. Rev. Lett. 77, 3835 (1996).

[10] C. Masciovecchio et al., Phys. Rev. Lett. 92, 247401 (2004).

[11] C. Masciovecchio et al., in Proceedings of 8th International Conference of Synchrotron Radiation Instrumentation, San Francisco, California, 2003 (American Institute of Physics, Warwick, 2004), p. 1190.

[12] G. Ruocco et al., Phys. Rev. Lett. 84, 5788 (2000).

[13] B. Fak and B. Dorner, Institute Laue Langevin (Grenoble, France) Technical Report No. 92FA008S, 1992.

[14] W. Dietsche and H. Kinder, Phys. Rev. Lett. 43, 1413 (1979).

[15] V. I. Kozub, A. M. Rudin, and H. R. Schober, Phys. Rev. B 50, 6032 (1994).

[16] A. R. Long, A. F. Cattell, and A. M. MacLeod, J. NonCryst. Solids 59-60, 1149 (1980); A. R. Long, A. C. Hanna, and A. M. MacLeod, J. Phys. C 19, 467 (1986).

[17] H. R. Philipp, in Handbook of Optical Constants of Solids, edited by D. Palik (Academic, New York, 1985).

[18] A. Goldoni et al. (to be published).

[19] A. Tanguy, J. P. Wittmer, F. Leonforte, and J. L. Barrat, Phys. Rev. B 66, 174205 (2002).

[20] B. Rossi, G. Viliani, E. Duval, L. Angelani, and W. Garber, Europhys. Lett. 71, 256 (2005).

[21] F. Leonforte, R. Boissiere, A. Tanguy, J. P. Wittmer, and J. L. Barrat, cond-mat/0505610.

[22] W. Schirmacher, Europhys. Lett. 73, 892 (2006).

[23] W. Schirmacher, G. Ruocco, and T. Scopigno (to be published).

[24] R. C. Zeller and P. O. Pohl, Phys. Rev. B 4, 2029 (1971).

[25] J. E. Graebner, B. Golding, and L. C. Allen, Phys. Rev. B 34, 5696 (1986). 